\title{
Precochlear Implant Assessment: Clinical Profile and Family History of Children with Severe Bilateral Prelingual Hearing Loss
}

\author{
Syed Hashim Raza ${ }^{1}$ Rehmana Waris ${ }^{1}$ Samina Akhtar ${ }^{2}$ Ramish Riaz ${ }^{3,4}$
}

${ }^{1}$ Children Hospital, Pakistan Institute of Medical Sciences, Islamabad, Islamabad, Pakistan

2 Department of Diagnostic Radiology, Pakistan Institute of Medical

Address for correspondence Ramish Riaz, PhD, Department of Diagnostic Radiology, Pakistan Institute of Medical Sciences, G-8, Islamabad, Islamabad, 44000, Pakistan

Sciences, Islamabad, Islamabad, Pakistan

3 Pakistan Institute of Medical Sciences \& Rawalpindi Medical College, (e-mail: ramish_exclusive@hotmail.com).

Islamabad, Islamabad, Pakistan

${ }^{4}$ Atta ur Rahman School of Applied Biosciences, National University of

Sciences and Technology, Islamabad, Islamabad, Pakistan

Int Arch Otorhinolaryngol 2020;24(4):e457-e461.

\begin{abstract}
Keywords

- hearing loss

- consanguinity

- cochlear implant

Introduction The prevalence of deafness is high in Pakistan. Knowledge regarding the clinical features of patients with profound hearing loss will not only help identify the cause but will also help in the strategic planning for public health interventions.

Objective The present study was conducted to cover in detail the clinical aspects of children with hearing loss, that is, age at presentation, associated deficits and disorders, possible cause of the disease, associated family history, and role of consanguineous marriage.

Methods The present study was performed from November 2016 to September 2018. All of the patients under 6 years of age with profound bilateral hearing loss who would benefit from cochlear implantation were included in the study. Detailed history was taken. The developmental skills were assessed for all areas, and the patients were scored regarding their motor, manipulative, visual, language, social and self-care skills according to the Schedule of Growing Skills II. Detailed family history was taken from the parents of the affected children. The Statistical Package for the Social Sciences (SPSS) software, version 20.0, was used for the statistical analysis.

Results The mean age of the children to be treated was $3.2 \pm 1.25$ years. Most patients (51.5\%) had a positive family history of disease. Consanguineous marriage was common; the parents of $76.9 \%$ of the patients were first-degree relatives. Most patients $(90.8 \%)$ had associated language impediments. In total, four (Ł3.07\%) patients had global developmental delay.

Conclusion Consanguineous marriage pattern plays an important role in diseases running in families. Development in these children is strongly linked to their age at the consultation.
\end{abstract}

received

August 17, 2019

accepted

November 3, 2019
DOI https://doi.org/

10.1055/s-0039-3402442.

ISSN 1809-9777.
Copyright $(2020$ by Thieme Revinter

Publicações Ltda, Rio de Janeiro, Brazil
License terms

(c) $(1) \$$ 


\section{Introduction}

Approximately 360 million people worldwide have disabling hearing loss, 32 million of whom are children. The prevalence of disabling hearing loss in children is highest in Southern Asia. ${ }^{1}$ The prevalence of profound bilateral hearing loss in Pakistan is 1.6 per 1,000 individuals. $^{2}$ There are around 150,000 congenitally deaf individuals in Pakistan. ${ }^{3}$

Congenital hearing loss could occur due to genetic causes, environmental factors, and prenatal factors, like congenital infections. However, the genetic causes account for the majority of the cases. ${ }^{4}$ Congenital deafness usually runs in families. Family history plays an important role in predicting the diseases and the genetic mutations associated with disease. ${ }^{5}$ Consanguineous marriage pattern is one of the important factors that increases the expression of recessive genetic disorders. High rates of consanguineous marriages result in an isolated genetic pool that increases the risk of developing rare diseases. ${ }^{2}$ Studies on congenital hearing loss have shown a high rate of consanguineous marriages among the parents of affected children. In such cases, more than one child is usually affected. ${ }^{6}$

Prelingual hearing loss is defined as loss of hearing before 2 years of age, that is, before the development of language skills. ${ }^{7}$ Most patients with prelingual hearing loss in industrialized nations have genetic defects. However, the cause of deafness in developing countries remains idiopathic. This could be due to the lack of facilities for genetic testing. Meningitis and use of ototoxic drugs are among the other few identifiable causes of prelingual hearing loss. ${ }^{8}$

Studies have shown that children with severe hearing impairment have multiple associated language and behavioral problems. ${ }^{9}$ Depression and stress disorders are also more common in deaf children as compared with their non-deaf peers. ${ }^{10}$ Language development is strongly linked to auditory perception. Cognitive development and social interactions are linked to the person's ability to speak. Prelingual hearing loss has a very negative effect on language development, which results in decreased cognitive skills and behavioral problems. Early identification of hearing loss and early intervention could increase the possibility of developing normal speech and language. ${ }^{11}$

Cochlear implantation can help deaf children in all aspects of learning and cognitive development. Studies have shown that better results are achieved in patients implanted before 3 years of age. ${ }^{8}$ Cochlear implantation was introduced in Pakistan in August 2000 through an international collaboration in a private setup at Lahore. However, due to the paucity of resources, only 52 patients were successfully implanted until 2005. Now, government hospitals have started to perform cochlear implantation at a comparatively lower cost. ${ }^{3}$ The present study is part of a project involving the first free cochlear implantation program in Pakistan. To date, there are very few studies conducted in Pakistan on hearing loss, especially on patients suitable for cochlear implantation. The present study was conducted to cover in detail the clinical aspects of children with hearing loss, that is, age at presentation, associated deficits and disorders, possible cause of the disease, associated family history, and role of consanguineous marriage in prelin- gual hearing loss. The present will be a baseline study that will provide details about the children visiting a public sector hospital in Pakistan for cochlear implantation. After the implantation, the present study will contribute to the comparison of outcomes between children with or without behavioral impediments.

\section{Materials and Methods}

The present descriptive study was performed from November 2016 to September 2018 after approval (Ref No:87-6-16) from the Children's Hospital. All of the patients under 6 years of age with profound bilateral hearing loss who would benefit from cochlear implantation were included in the study. The patients associated with other syndromes were excluded. A detailed clinical evaluation was performed. The patients were scored regarding their motor, manipulative, visual, language, social and self-care skills according to the Schedule of Growing Skills II. Radiological investigations such as magnetic resonance imaging and computed tomography scans were also observed. Detailed family history was taken from the parents of the affected children. The Statistical Package for the Social Sciences (SPSS, IBM Corp., Armonk, NY, US) software, version 20.0, was used for the statistical analysis. At first, the data was arranged and values were assigned to them. Odds ratio (OR), relative risk (RR) and Spearman correlation (r) tests were applied to check the associations. Dispersion in the quantitative data was analyzed by standard deviation. Values of $p>0.05$ were set as statistically significant.

\section{Results}

The mean age of the children during the treatment was $3.2 \pm 1.25$ years. The mean age at the diagnosis was $14 \pm 9.4$ months. In total, $70 \%$ of the patients were male, and most were toddlers (- Figure $\mathbf{1}$ ).

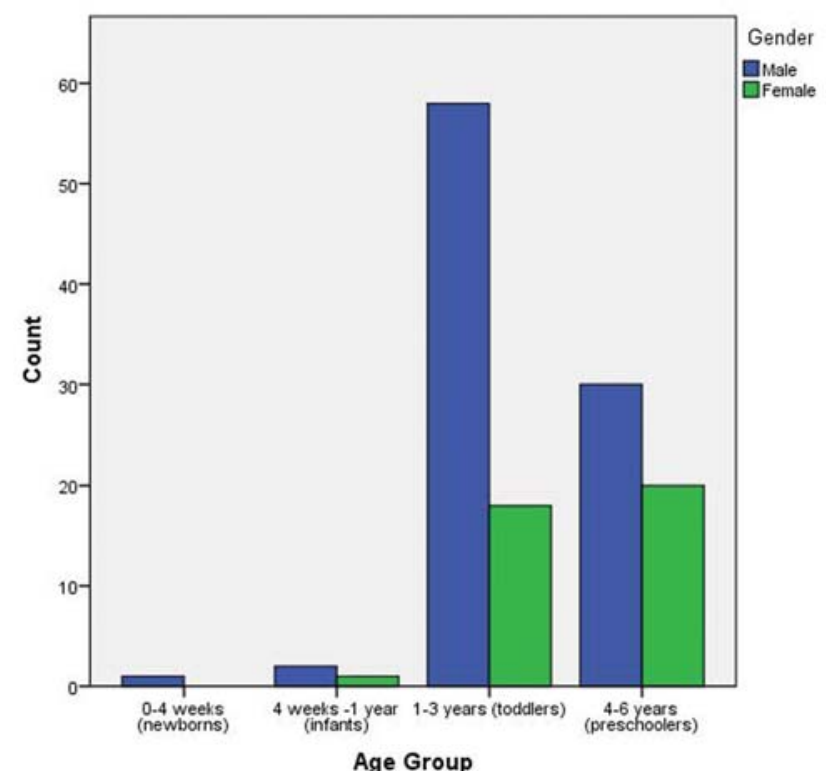

Fig. 1 Distribution of patients according to age and gender. 
Table 1 Relation of family member affected and consanguineous marriage

\begin{tabular}{|c|c|c|c|c|}
\hline & & \multicolumn{2}{|c|}{$\begin{array}{l}\text { Consanguineous } \\
\text { marriage }\end{array}$} & \multirow[t]{2}{*}{ Total } \\
\hline & & Yes & No & \\
\hline \multirow{7}{*}{$\begin{array}{l}\text { Relation of } \\
\text { family member }\end{array}$} & No family history of disease & 42 & 21 & 63 \\
\hline & Brother affected & 13 & 0 & 13 \\
\hline & Sister affected & 11 & 0 & 11 \\
\hline & First-degree relative affected & 9 & 6 & 15 \\
\hline & More than one family member affected & 4 & 1 & 5 \\
\hline & Both sibling and one first-degree relative affected & 3 & 0 & 3 \\
\hline & More than three first-degree relatives affected & 18 & 2 & 20 \\
\hline \multicolumn{2}{|l|}{ Total } & 100 & 30 & 130 \\
\hline
\end{tabular}

Most patients (51.5\%) had positive family history of disease. Consanguineous marriage was common; the parents of $76.9 \%$ ( $n=100)$ of the patients were first-degree relatives. A significant association was found between consanguineous marriage pattern ( $\mathrm{OR}=3.22 ; \mathrm{RR}=1.9 ; p=0.008)$ and positive family history. With the detailed evaluation, we noted that, among the consanguineous group, immediate family members were affected, that is, siblings, while, in the other group, there was positive history in first-degree relatives ( - Table $\mathbf{1}$ ).

In total, $70 \%$ of the patients had been born at full term by spontaneous natural delivery. Only $23 \%(n=30)$ had been born via cesarian section, and, among those, 1 birth had been premature. Most patients $(n=105 ; 81 \%)$ did not have any issues at birth or during the postnatal period. Among those with previous history of diseases, seizure $(n=8,32 \%)$ was the most $(n=4,16 \%)$, sepsis ( $n=4,16 \%)$, neonatal severe jaundice $(n=3,12 \%)$, heart disease $(n=3,12 \%)$, tuberculosis ( $n=1,4 \%)$, severe diarrhea $(n=1,4 \%)$ and premature birth $(n=1,4 \%)$. Moreover, patients with seizures and meningitis were found to have negative family history for disease. Most patients $(n=79 ; 61 \%)$ had weights below the 10 th percentile. The majority of the sample had ( $n=115 ; 88.4 \%)$ had occipital-frontal circumference (OFC) below the 10th percentile. Positive radiological findings were only observed in 25 (20\%) patients, cerebral atrophy was $n=9,6.92 \%$ ) finding, followed by demyelination $n=4,3.07 \%$ ), arachnoid cyst ( $n=3,2.30 \%$ ), fluid in the mastoid ( $n=3,2.3 \%), n=2,1.53 \%$ ), otomastoiditis ( $n=1,0.76 \%)$, hyperintense globus pallidus $(n=1,0.76 \%)$, epidermoid cyst $(n=1,0.76 \%)$, and tuberous sclerosis $(n=1,0.76 \%)$.

Most patients ( $n=118 ; 90.8 \%$ ) had associated language impediments, and 66 (50.8\%) of those had severely impaired speech and language skills. Impaired language skills were found to be strongly associated $(r=0.64 ; p>0.001)$ with late start of consultation at the hospital. The majority of the patients with severe impairment belonged to the preschool age group, that is, 4 to 5 years of age (-Table $\mathbf{2}$ ).

Among other growing skills, visual skills were impaired in 11 patients ( $8.46 \%)$, followed by impaired cognitive skills in 8 patients (6.15\%), impaired self-care skills in 7 patients (5.38\%), impaired interactive and social skills in 7 patients (5.38\%), manipulative skills in 6 patients (4.6\%), and gross motor skills in 4 patients (3.07\%) (-Figure 2). A total of four patients had global developmental delay, and two of them had autism spectrum disorder.

All of the patients with cognitive delay had OFC below the 10th percentile. The detailed analysis revealed that all of the patients with cognitive, social or self-care impairments had associated moderate or severe language impairment.

\section{Discussion}

The prevalence of deafness is high in Pakistan. Results from an epidemiological study ${ }^{12}$ conducted in the country's largest province, Punjab, showed a prevalence of 20 cases per every 10,000 individuals, with consanguinity being a major

Table 2 Age Group and Speech and Language Skills

\begin{tabular}{|c|c|c|c|c|c|c|c|}
\hline & & \multicolumn{5}{|c|}{ Speech and language } & \multirow[t]{2}{*}{ Total } \\
\hline & & Normal & Acceptable & $\begin{array}{l}\text { Mild } \\
\text { impairment }\end{array}$ & $\begin{array}{l}\text { Moderate } \\
\text { impairment }\end{array}$ & $\begin{array}{l}\text { Severe } \\
\text { impairment }\end{array}$ & \\
\hline \multirow[t]{4}{*}{ Age group } & 0-4 weeks (newborns) & 0 & 0 & 1 & 0 & 0 & 1 \\
\hline & 4 weeks to 11 months (infants) & 3 & 0 & 0 & 0 & 0 & 3 \\
\hline & $1-3$ years (toddlers) & 9 & 7 & 14 & 23 & 23 & 76 \\
\hline & 4-6 years (preschoolers) & 0 & 0 & 0 & 7 & 43 & 50 \\
\hline \multicolumn{2}{|l|}{ Total } & 12 & 7 & 15 & 30 & 66 & 130 \\
\hline
\end{tabular}




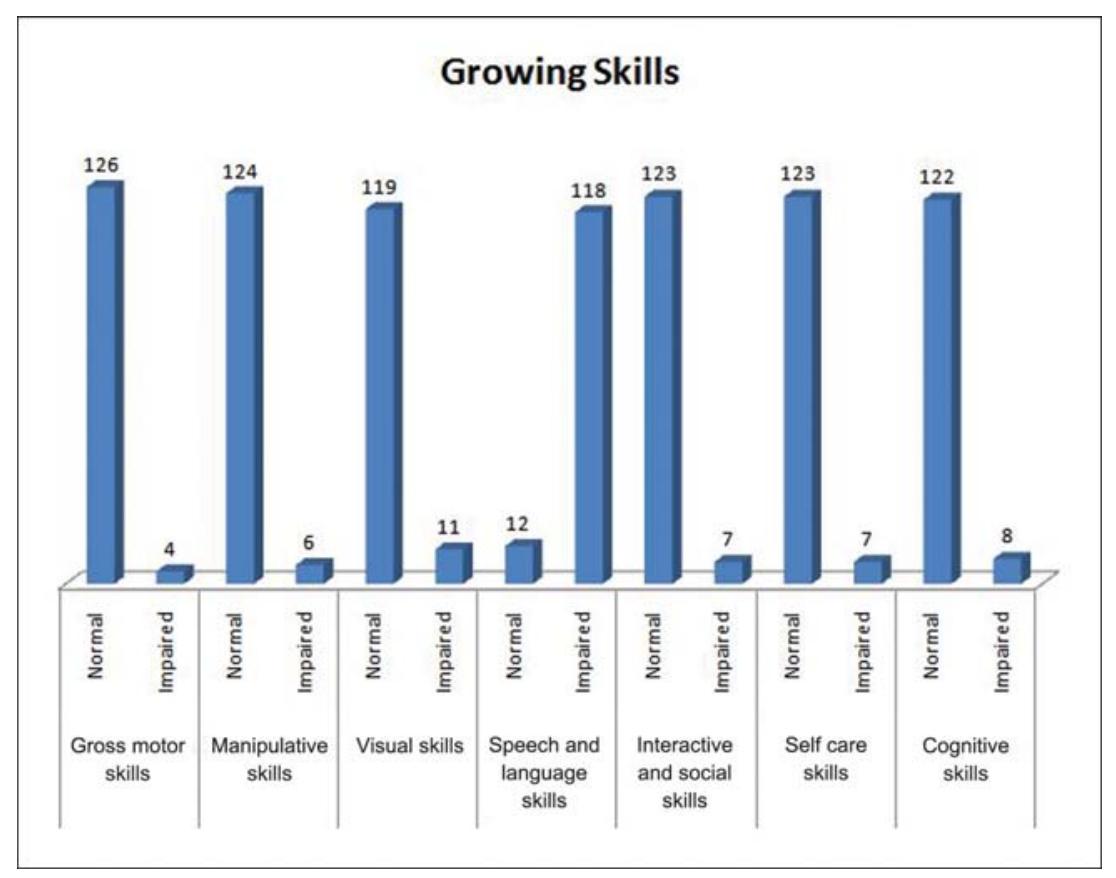

Fig. 2 Graph showing growing skills among the patients.

cause behind it. Knowledge regarding the clinical features and characteristics of patients with profound hearing loss will not only help identify the cause but will also contribute to the strategic planning for public health interventions.

Cochlear implantation has significantly improved the quality of life of deaf children. The outcome tends to be better in children than in adults. ${ }^{7}$ However, the procedure is costly. In Pakistan, cochlear implantation was first introduced in 2000; however due to the high cost, only 55 patients were implanted until $2005 .{ }^{3}$ Since 2016 , the government has taken an initiative to provide free transplants to affected children. The present study is a part of that project.

The mean age at the diagnosis was 14 months; however, the mean age at the consultation for cochlear implant assessment was 3.2 years. This shows the lack of a proper health care system in terms of referral. Moreover, studies conducted in the West have shown better speech and language outcomes in patients implanted before the development of proper speech that is, from the ages of 12 months to 3 years, than in those implanted at the ages of 4 or $5 .^{13}$

Positive family history and consanguinity are important risk factors for profound hearing loss. Our results were also in line with this. A study ${ }^{6}$ conducted in Oman showed that $70 \%$ of the parents of affected children had a consanguineous marriage pattern; out of these, $67.6 \%$ also had affected siblings. Our results also showed similar findings.

Genetic factors are the most common identifiable cause of deafness. ${ }^{14}$ Due to the high cost and lack of genetic laboratories, we did not perform a genetic analysis. However, the presence of family history and more than one family member with a disease provide a strong clue regarding genetic involvement in these families. Other causes involve infections, such as meningitis, otitis media and the mumps. Premature birth, neonatal jaundice and ototoxic drugs are also among the major contributing risk factors. ${ }^{14}$ In the present study, most patients had weight below the 10th percentile. However, most of them did not present any identifiable causes, that is, had no history of disease. Among those with positive history, seizures and meningitis were the most common complaints.

The child's ability to speak is strongly linked to their ability to hear; therefore, most deaf children are also unable to speak. Delay in language development results in many behavioral disorders. ${ }^{9}$ A study ${ }^{15}$ conducted in Pakistan on special children and deaf children showed that deaf children, due to their lack of speaking ability, have low perception of their self-image, and are unable to carry out their routine activities effectively. They have higher rates of stress, depression and anxiety disorders. Unfortunately, society also isolates such children, which results in psychological disorders. Such children show delayed cognitive and interactive social skills. In the present study $(90 \%, n=117)$ of the patients had associated language delays; out of these, (50\%, $n=59)$ had severe language impediment. Cognitive and social developments were more delayed among children with severe language impairment, and language development was more severely affected in children presenting late to the hospital.

\section{Conclusion}

Profound bilateral hearing loss is a multifaceted condition with multiple social and medical aspects. Developmental delays are not uncommon in children with profound hearing loss, and are strongly linked to their age at the consultation. Effective public health measures and awareness would lead to early diagnosis and early intervention, which in turn, will lead to better outcomes and better quality of life for these children. The present study also depicts the role of consanguineous marriage pattern and disease running in families. Public awareness 
regarding consanguinity and the risk of genetic disorders, including deafness, will help reduce the rate of disease. A study performed with these children after cochlear implantation will help in the determination of the outcomes and of the association between these factors. Future studies should be conducted on the involvement of genes in hearing loss; this will not only help find the culprit gene but will also open the doors to treat hearing loss at the genetic level.

\section{Conflict of Interests}

The authors have no conflict of interests to declare.

\section{References}

1 World Health Organization. WHO global estimates on prevalence of hearing loss. World Health Organization; 2012

2 Ali G. Genetic deafness in Pakistani population. J Pak Med Assoc 2010;60(06):418-419

3 Khan MI, Mukhtar N, Saeed SR, Ramsden RT. The Pakistan (Lahore) cochlear implant programme: issues relating to implantation in a developing country. J Laryngol Otol 2007;121(08): $745-750$

4 Korver AM, Smith RJ, Van Camp G, et al. Congenital hearing loss. Nat Rev Dis Primers 2017;3(03):16094

5 McMahon CM, Kifley A, Rochtchina E, Newall P, Mitchell P. The contribution of family history to hearing loss in an older population. Ear Hear 2008;29(04):578-584
6 Al Khabori M. Causes of severe to profound deafness in Omani paediatric population. Int J Pediatr Otorhinolaryngol 2004;68(10): 1307-1313

7 Leal AF. Screening of Patients for Cochlear Implant Through a Questionnaire Online. Group Profile of Patients Pre-and Peri lingual Not Summoned. Int Arch Otorhinolaryngol 2010;14(02):184-191

8 Barbosa MH, Felix F, Ribeiro MG, Tomita S, Pinheiro C, Baptista MM. Profile of patients assessed for cochlear implants. Rev Bras Otorrinolaringol (Engl Ed) 2014;80(04):305-310

9 Bigler D, Burke K, Laureano N, Alfonso K, Jacobs J, Bush ML. Assessment and Treatment of Behavioral Disorders in Children with Hearing Loss: A Systematic Review. Otolaryngol Head Neck Surg 2019;160(01):36-48

10 Hall WC. What you don't know can hurt you: The risk of language deprivation by impairing sign language development in deaf children. Matern Child Health J 2017;21(05):961-965

11 Shojaei E, Jafari Z, Gholami M. Effect of Early Intervention on Language Development in Hearing-Impaired Children. Iran J Otorhinolaryngol 2016;28(84):13-21

12 Ullah S, Aslamkhan M, Ali A, Idrees M. Causes of deafness in the Punjab region of Pakistan and the role of consanguinity. Public Health 2017;145:93-95

13 Krug E, Cieza A, Chadha S, et al. Childhood Hearing Loss: Strategies for prevention and care. Switzerland: World Health Organization; 2016:6-7

14 Akram B, Bashir R. Special Education and Deaf Children in Pakistan: An Overview. Journal of Elementary Education. 2012;22(02):33-34

15 Schafer E, Utrup A. The Effect of Age of Cochlear Implantation on Speech Intelligibility to Others. JEPRA 2016;22:1-11 